\title{
TRK Fusion Cancer: Patient Characteristics and Survival Analysis in the Real-World Setting
}

\author{
Lyudmila Bazhenova $^{1} \cdot$ Andrew Lokker $^{2} \cdot$ Jeremy Snider $^{2} \cdot$ Emily Castellanos $^{2} \cdot$ Virginia Fisher $^{3} \cdot$ Marc Fellous $^{4}$. \\ Shivani Nanda ${ }^{4} \cdot$ Jihong Zong ${ }^{4} \cdot$ Karen Keating $^{4} \cdot$ Xiaolong Jiao $^{4}$
}

Accepted: 3 February 2021

(c) The Author(s) 2021

\begin{abstract}
Background Neurotrophic tyrosine receptor kinase (NTRK) gene fusions are oncogenic drivers in various tumor types. While $N T R K$ gene fusions are predictive of benefit from tropomyosin receptor kinase inhibitors regardless of tumor type, the prognostic significance of $N T R K$ gene fusions in a pan-tumor setting remains unclear.

Objective This study evaluated the characteristics and prognosis of tropomyosin receptor kinase fusion cancer in the realworld setting.

Patients and Methods This retrospective study used a de-identified clinico-genomic database and included patients with cancer who had comprehensive genomic profiling between January 2011 and July 2018. Patients were classified as having cancer with NTRK gene fusions or NTRK wild-type genes. Patients were matched with a 1:4 ratio (NTRK fusion:NTRK wild-type) using the Mahalanobis distance method on demographic and clinical characteristics, including age and Eastern Cooperative Oncology Group performance status. Descriptive analysis of clinical and molecular characteristics was conducted. Kaplan-Meier estimator and Cox regression were used for overall survival analysis.

Results Median overall survival was 12.5 months (95\% confidence interval 9.5-not estimable) and 16.5 months (95\% confidence interval 12.5-22.5) in the NTRK gene fusion $(n=27)$ and NTRK wild-type cohorts $(n=107)$, respectively (hazard ratio 1.44; $95 \%$ confidence interval $0.61-3.37 ; p=0.648$ ). Co-occurrence of select targetable biomarkers including $A L K$, $B R A F, E R B B 2, E G F R, R O S 1$, and KRAS was lower in cancers with NTRK gene fusions than in NTRK wild-type cancers.

Conclusions Although the hazard ratio for overall survival suggested a higher risk of death for patients with NTRK gene fusions, the difference was not statistically significant. Co-occurrence of NTRK gene fusions and other actionable biomarkers was uncommon.
\end{abstract}

Lyudmila Bazhenova

lbazhenova@health.ucsd.edu

Xiaolong Jiao

xiaolong.jiao@bayer.com

1 Moores Cancer Center, University of California San Diego, San Diego, 3855 Health Sciences Drive, La Jolla, CA 92037, USA

2 Flatiron Health, Inc., New York, NY, USA

3 Foundation Medicine, Inc., Cambridge, MA, USA

4 Bayer HealthCare Pharmaceuticals, Inc., 100 Bayer Blvd, Whippany, NJ 07981, USA

\section{Key Points}

Neurotrophic tyrosine receptor kinase (NTRK) gene fusions rarely co-occur with other known oncogenic alterations and in tumors that harbor them they are the primary oncogenic drivers.

$N T R K$ gene fusions are targetable oncogenic alterations and thus the adoption of widespread screening is recommended especially for patients with rare tumor types.

Based on current evidence, patients with NTRK gene fusions do not have a statistically worse or better prognosis than patients with NTRK wild-type tumors. 


\section{Introduction}

The tropomyosin receptor kinase (TRK) family of receptors comprises TRKA, TRKB, and TRKC, which are encoded by the neurotrophic tyrosine receptor kinase (NTRK) genes NTRK1, NTRK2, and NTRK3, respectively $[1,2]$. TRK receptors are important for the regulation of pain and body temperature $[3,4]$, appetite control, learning [5-7], proprioception, memory, and mood [8].

Recurrent NTRK gene fusions have been reported as oncogenic drivers in a wide variety of adult and pediatric tumor types [1]. NTRK gene fusions occur when the $3^{\prime}$ region of the NTRK gene, encoding the tyrosine kinase domain, is joined with the $5^{\prime}$ end of a fusion partner gene, either by an intra- or inter-chromosomal rearrangement [9]. The resulting fusion gene leads to the expression of a chimeric protein with constitutively active or overexpressed kinase function that drives downstream signaling to promote tumor growth and survival [9]. In addition, a variety of other NTRK gene alterations have been identified in cancer, although their role in tumorigenesis is unclear.

NTRK gene fusions are found at high frequencies (up to or greater than $90 \%$ ) in selected rare tumor types (secretory breast carcinoma, salivary gland secretory carcinoma, congenital mesoblastic nephroma, and infantile fibrosarcoma) and at lower frequencies (generally $<1 \%$ ) in a range of other tumor types, including lung and colorectal cancers [2].

For patients with tumors that harbor NTRK gene fusions, TRK inhibitors provide an effective treatment option, as demonstrated in several clinical trials [10]. Larotrectinib is a first-in-class, highly selective, central nervous systemactive, small-molecule TRK inhibitor that was first approved by the US Food and Drug Administration in November 2018 and received conditional approval in Europe in September 2019 for the treatment of adult and pediatric patients with solid tumors harboring an NTRK gene fusion [11-13]. Larotrectinib has demonstrated a high and durable objective response rate in a pooled analysis of three phase I/II trials in adults and/or children (NCT02122913, NCT02637687, NCT02576431) that included 159 patients with 17 different tumor types. Investigator-assessed objective response rate was $79 \%$ (95\% confidence interval [CI] 72-85), regardless of tumor type and age [13]. Median duration of response was 35.2 months (95\% CI 22.8-not estimable) [13]. Entrectinib is a multi-kinase inhibitor [14] that targets $A L K, R O S 1$, and NTRK $1 / 2 / 3$ and is approved by the Food and Drug Administration for adult and pediatric patients aged $\geq 12$ years with locally advanced or metastatic NTRK fusion-positive solid tumors [15]. In a pooled subgroup analysis of 54 patients, the objective response rate was $57 \%$ (95\% CI 43.2-70.8) [16]. Median duration of response was 10.4 months $(95 \%$ CI 7.1-not estimable) [16].
Although there has been significant progress in treating TRK fusion cancer, several important questions remain unanswered. There are currently very limited data on the demographic, clinical, and genomic characteristics as well as natural histories of patients whose tumors harbor an NTRK gene fusion compared with those whose tumors do not, especially from the real-world setting [17]. NTRK gene fusions occur in diverse tumor types with different natural histories. Therefore, understanding the natural history of TRK fusion cancer as a single entity is challenging. Single-arm studies of TRK inhibitors have shown impressive results compared with historical data for standard-of-care therapies. However, the prognosis of patients with cancer harboring NTRK gene fusions compared with similar patients with NTRK wild-type cancer is unknown.

To address these questions, we conducted a retrospective matched cohort study to investigate demographic, clinical, and molecular characteristics, as well as survival in patients with cancer with and without $N T R K$ gene fusions, using a US de-identified real-world clinico-genomic database comprising clinical data from electronic health records that was linked to comprehensive genomic profiling (CGP) data obtained in the process of routine clinical care.

\section{Methods}

\subsection{Study Design}

A retrospective study was conducted in patients with solid malignancies from the de-identified Flatiron Health-Foundation Medicine clinico-genomic database (CGDB; version November 2018) whose tumors had been tested by CGP between January 2011 and July 2018. The CGDB includes data collected routinely during patient healthcare, and is generated by linking real-world clinical data from the Flatiron Health electronic health record-derived, de-identified database, including data on clinical and demographic characteristics, treatment patterns and outcomes, with genomic data from the Foundation Medicine database through a deterministic matching process [18]. Real-world clinical data from electronic health records, including data on clinical and demographic characteristics, treatment patterns, and outcomes, were sourced from Flatiron Health's nationwide database. At the time of this study, the database contained data from $>280$ cancer clinics across $\sim 800$ sites in the USA, representing approximately 2 million patients with cancer from both community and academic sites. Genomic data from Foundation Medicine CGP tests contained somatic mutations, copy number alterations and rearrangements, complex biomarkers including tumor mutational burden (TMB), and microsatellite instability (MSI) status reported from Foundation Medicine's genomic profiling assays 
FoundationOne ${ }^{\circledR}$, FoundationOne Heme, and FoundationOne CDx [19]. Over 300 genes were sequenced; the full coding sequences of the genes and select introns from genes often rearranged or altered in cancer (including $A L K, B R A F$, $E R B B 2, E G F R, R O S 1$, and $K R A S$ ) were analyzed. In a validation study of the FoundationOne ${ }^{\circledR}$ NGS assay, sensitivity was $>99 \%$ for base substitutions, $98 \%$ for indels, and $>95 \%$ for copy number alterations. The assay demonstrated high specificity, with a positive predictive value of alteration calls of over 99\% [19]. This assay also demonstrated strong concordance with a validated RNA NGS fusion panel for the detection of NTRK gene fusions [20]. Furthermore, the assay recently received Food and Drug Administration approval as a companion diagnostic for larotrectinib [21, 22].

\subsection{Eligibility Criteria}

Eligible patients had at least one documented clinical visit in the Flatiron Health network between 1 January, 2011 and 1 August, 2018 and underwent CGP by Foundation Medicine prior to 1 August, 2018 using a version of one of the solid tumor (FoundationOne and FoundationOne CDx) or hematologic (FoundationOne Heme) assays. Foundation Medicine began CGP in August 2012; therefore, any patient with a death date before the start of 2012 was deemed to be included in the database by error and excluded from the study to avoid any data issues.

\subsection{Molecular Classifications and Covariate Matching}

During the CGP period of the study, FoundationOne assays used an evolving set of baits and an evolving computational pipeline for the detection of NTRK1, NTRK2, and NTRK3. All bait sets had complete coverage of the coding exons of NTRK1, NTRK2, and NTRK3; NTRK1, NTRK2, and ETV6 intron coverage varied by assay (Table 1 of the Electronic Supplementary Material [ESM]).

Patients were classified into two cohorts (Fig. 1): patients whose tumors had an NTRK gene fusion (Cohort 1) and patients with the same tumor types seen in the NTRK gene fusion cohort but without any NTRK gene alteration of known or likely functional significance (including fusions, point mutations, other rearrangements, amplifications, and deletions; Cohort 2, NTRK wild-type non-matched). A subgroup of the Cohort 2 patients was then matched with patients with cancer harboring NTRK gene fusions based on baseline patient characteristics (Cohort 2, NTRK wildtype matched). Matching of patients within histologies was conducted using the Mahalanobis distance method [23], with a ratio of 1:4 (NTRK fusion:NTRK wild-type), except where limited by sample size (there were only three patients with NTRK wild-type follicular dendritic cell sarcomas, and none with salivary gland secretory carcinoma). In this study, the Mahalanobis distance method was considered preferable over other regression-based propensity score methods to define patient similarity, to avoid biases caused by the outsized effect of the patient characteristics seen in more common cancers, such as non-small cell lung cancer and colorectal cancer, that might arise from a regression-based approach. In addition, the Mahalanobis distance method is well suited to cases where there is an interest in matching on a small number of covariates [24]. Matching was also viewed as a suitable approach as there were generally a very large number of potential wild-type matches available. The matching was done on the following demographic and clinical characteristics: age at time of CGP report, albumin $(<3.5 \mathrm{~g} / \mathrm{dL}$ vs $>3.5 \mathrm{~g} / \mathrm{dL})$, documented antineoplastic use, closest documented Eastern Cooperative Oncology Group (ECOG) performance status from 30 days before to 7 days after index date, practice type (academic vs community), and year of CGP report. Missing ECOG performance status and serum albumin values were considered distinct categories for the matching analysis. Patient ages $>85$ years were masked for de-identification reasons. Guidelines indicate that a threshold of 0.1 or 0.25 for the Mahalanobis distance represents reasonable cut-offs for matching of baseline covariates [25]. In this study, a threshold of 0.1 was used, as an absolute mean difference of less than 0.1 indicates a negligible difference between groups [26].

\subsection{Objectives and Statistical Analysis}

The primary objective of this study was to evaluate overall survival (OS) from the date of the CGP report for patients with cancer harboring NTRK gene fusions and patients with cancer with wild-type NTRK genes. Secondary objectives included description of patient characteristics, treatment patterns, and genomic alterations in patients with NTRK gene fusions and in patients with NTRK wild-type genes.

Descriptive analysis of patient characteristics was conducted. Frequencies were provided for categorical variables, while means, standard deviations, medians, and ranges were provided for continuous variables. The Kaplan-Meier method and Cox regression were used for OS analysis, with the date of the CGP report as the index date. Patients who did not die were censored at the last activity date. Mortality data were extracted from the electronic health record structured data field "patient date of death", if available; otherwise, the patient's last activity date was used [27]. Medians and 95\% CIs were calculated for OS. Unadjusted hazard ratios (HRs) and the associated 95\% CIs were also calculated.

The presence of NTRK gene fusions and co-occurrence of the following biomarkers, which are considered clinically actionable (i.e., with an associated targeted therapy 
or potential for off-label targeted therapy) or of emerging research interest, was determined: TMB, MSI, and oncogenic functional alterations in $A L K, B R A F, E R B B 2, E G F R$, ROS1, and KRAS [28].

\section{Results}

\subsection{Patient Characteristics}

Among 33,429 eligible patients in the CGDB with solid tumors, 29 patients had tumors harboring an NTRK gene fusion (Cohort 1). The evaluable dataset comprised 15,971 patients with a tumor type represented in patients with NTRK gene fusion. This dataset contained 12 distinct tumor or histology types, the most common of which were colorectal cancer, salivary gland cancer, lung cancer, and sarcomas.

The evaluable 15,971 patients were subsequently filtered by NTRK alteration status; 12,456 had no NTRK gene alterations of known or likely functional significance (Cohort 2, all: NTRK wild-type). The remaining 3515 patients had $N T R K$ gene alterations of known or likely functional significance excluding gene fusions or rearrangements. The CGP assays used to identify each cohort are described in Table 1 of the ESM. After covariate matching of demographic and clinical characteristics using the Mahalanobis distance method within each tumor type, the matched NTRK wildtype cohort (Cohort 2: NTRK wild-type matched) contained 107 patients (Fig. 1). This was fewer than the 116 patients anticipated (based on 1:4 matching with Cohort 1) because of insufficient patients with salivary gland secretory carcinomas to produce a 1:4 match in Cohort 2, and patients with mismatched baseline covariates. After covariate matching, the mean difference between cohorts for each covariate was below the 0.1 threshold, except for age (Fig. 1 of the ESM).

Of the 29 patients included in Cohort 1 (NTRK fusion), $16(55 \%)$ were female and 13 (45\%) were male, the median age was 60 years (range 49-65 years), and the majority were Caucasian $(n=20 ; 69 \%)$. A total of 12 tumor types were identified in the NTRK fusion cohort, with the most common being colorectal cancer $(n=7)$, salivary gland cancer $(n=5)$, lung cancer $(n=4)$, and sarcomas $(n=4)$. Baseline characteristics were similar in Cohort 2 (NTRK wild-type non-matched, $n=12,349)$, in which 6785 patients $(55 \%)$ were female, the median age was 64 years (range 55-72 years), and 8379 patients (68\%) were Caucasian. The most common tumor types in this cohort were lung cancer $(n=4133)$, colorectal cancer $(n=3927)$, and breast carcinoma $(n=1313)$. Within the matched subset of the NTRK wild-type cohort ( $n=107), 62$ patients $(58 \%)$ were female, the median age was 63 years (range 55-70 years), and 76 patients $(71 \%)$ were Caucasian. In both the NTRK fusion and wild-type cohorts, the majority of patients were identified from a community practice (Table 1 ).

A majority of patients received at least one antineoplastic therapy in the NTRK fusion, NTRK wild-type non-matched, and NTRK wild-type matched cohorts $(55 \%, 76 \%$, and $66 \%$, respectively). The proportion of patients who received any programmed death-ligand 1 therapy was lower in the NTRK fusion cohort than both the NTRK wild-type non-matched and matched cohorts (3\% vs $16 \%$ and $8 \%$, respectively; Table 1).

In the NTRK fusion cohort, fusions occurred more frequently in NTRK1, reported in 18 patients $(62 \%)$, than in NTRK2 and NTRK3, reported in three patients (10\%) and eight patients (28\%), respectively. A total of 15 different $N T R K$ fusion partners were identified; the most frequently detected fusions were ETV6-NTRK3 $(n=8), T P M 3-N T R K 1$ $(n=6)$, and TPR-NTRK1 $(n=3)$ [Table 2]. At the time of this report, seven of these were novel fusion partners based on our knowledge.

\subsection{Survival Analysis}

Twenty-seven patients from the NTRK fusion cohort were matched with 107 patients in the NTRK wild-type cohort for the OS analysis. Patients with salivary gland secretory carcinoma in the NTRK fusion cohort $(n=2)$ were excluded from the OS analysis because of a lack of patients with this tumor type in the NTRK wild-type cohort. Treatments and outcomes that occurred outside of the clinical setting or were not documented in the patient's record were not included. The median follow-up was similar across both cohorts, 7.5 months (95\% CI 2.2-12.5) in the NTRK fusion cohort and 7.6 months (95\% CI 2.2-16.2) in the NTRK wildtype cohort. In an unadjusted analysis, median OS from the CGP report date of 12.5 months (95\% CI 9.5-not evaluable) and 16.5 months (95\% CI 12.5-22.5) was observed in the NTRK fusion cohort and covariate-matched NTRK wild-type cohort, respectively, with a HR of 1.44 (95\% CI 0.61-3.37; $p=0.648$ ) (Fig. 2).

\subsection{Biomarkers}

In the NTRK fusion cohort, five patients (17\%) had cooccurring genomic alterations in BRAF, EGFR, or KRAS. No patients had $A L K$ rearrangements, $E R B B 2$ amplifications, or ROS1 alterations. High TMB ( $\geq 20 \mathrm{mut} / \mathrm{mB}$ ) was the most frequent co-occurring biomarker in the NTRK fusion cohort, detected in six patients (21\%; Table 3). High MSI was reported in the colorectal cancer group only $(3 / 4 ; 75 \%$; Table 4).

In the NTRK wild-type cohort, known oncogenic alterations were reported in 7296 patients (59\%) in the non-matched group and 44 patients $(41 \%)$ in the matched 
Table 1 Baseline characteristics

Cohort 1: NTRK fusion Cohort 2: NTRK wild-type $(n=12,456)$

$(n=29)$

Non-matched $(n=12,349)$

Matched $(n=107)$

Patient characteristics

Sex, $n(\%)$

Female

Male

Age, median (range), years

$6(55.2)$

$6785(54.9)$

$62(57.9)$

$13(44.8)$

$5563(45.0)$

$45(42.1)$

$60(49-65)$

$64(55-72)$

$63(55-70)$

Race/ethnicity, $n(\%)$

Asian

0

Black/African American

$1(3.5)$

Hispanic/Latino

White/Caucasian

0

$20(69.0)$

Other

$1(3.5)$

Missing

$7(24.1)$

Primary tumor type, $n(\%)$

Colorectal cancer

Salivary gland

Lung

Sarcoma

Pancreatic

Brain medulloblastoma

Breast carcinoma

Melanoma

Thyroid carcinoma

Unknown primary neuroendocrine tumor

Unknown primary carcinoma NOS

Unknown primary adenocarcinoma

ECOG performance status, ${ }^{a} n(\%)$

0

1

$\geq 2$

Missing

7 (24.1)

$5(17.2)$

$4(13.8)$

$4(13.8)$

$2(6.9)$

$1(3.4)$

$1(3.4)$

$1(3.4)$

$1(3.4)$

$1(3.4)$

$1(3.4)$

$1(3.4)$

$3(10.3)$

$2(6.9)$

0

$24(82.8)$

Practice type, $n(\%)$

Academic

$6(20.7)$

Community

$23(79.3)$

CGP assay type

FoundationOne ${ }^{\circledR}$

FoundationOne CDx

FoundationOne Heme

PD-L1 status at CGP report, $n(\%)$

High $(>50)$

Low (1-50)

Negative $(<1)$

Missing

Serum albumin high vs low, ${ }^{\mathrm{b}} n(\%)$

Serum albumin, g/dL, $\leq 3.5$

Serum albumin, g/dL, $>3.5$

Missing

Serum albumin, g/dL, mean (range)
$24(82.8)$

$1(3.5)$

$4(13.8)$

0

$2(6.9)$

$1(3.5)$

$26(89.7)$

$2(6.9)$

$13(44.8)$

14 (48.3)

$4.0(3.6-4.3)$
$291(2.4)$

$1(0.9)$

775 (6.3)

$36(0.3)$

$6(5.6)$

0

8379 (67.9)

$1106(9.0)$

$1762(14.3)$

$3927(31.8)$

$82(0.7)$

4133 (33.5)

204 (1.7)

$1068(8.6)$

$22(0.2)$

1313 (10.6)

317 (2.6)

$48(0.4)$

$20(0.2)$

394 (3.2)

821 (6.6)

2074 (16.8)

2990 (24.2)

1131 (9.2)

6154 (49.8)

1346 (10.9)

11,003 (89.1)

11,754 (95.2)

492 (4.0)

210 (1.7)

255 (2.1)

287 (2.3)

$1272(10.3)$

10,535 (85.3)

1839 (14.9)

5860 (47.5)

4650 (37.7)

$3.80(3.5-4.1)$
76 (71.0)

9 (8.4)

15 (14.0)

28 (26.2)

12 (11.2)

$16(15.0)$

15 (14.0)

8 (7.5)

4 (3.7)

4 (3.7)

4 (3.7)

4 (3.7)

4 (3.7)

4 (3.7)

4 (3.7)

12 (11.2)

12 (11.2)

0

83 (77.6)

21 (19.6)

$86(80.4)$

94 (87.9)

3 (2.8)

$10(9.3)$

0

$1(0.9)$

9 (8.4)

97 (90.7)

5 (4.7)

48 (44.9)

54 (50.5)

$4.0(3.9-4.3)$ 
Table 1 (continued)

\begin{tabular}{|c|c|c|c|}
\hline & \multirow{2}{*}{$\begin{array}{l}\text { Cohort } 1: \text { NTRK fusion } \\
(n=29)\end{array}$} & \multicolumn{2}{|c|}{ Cohort 2: NTRK wild-type $(n=12,456)$} \\
\hline & & Non-matched $(n=12,349)$ & Matched $(n=107)$ \\
\hline Absolute neutrophil count $\left(10^{9} / \mathrm{L}\right),{ }^{\mathrm{b}}$ mean (range) & $5.2(3.1-7.1)$ & $4.4(2.8-6.6)$ & $4.1(3.0-5.8)$ \\
\hline Missing, $n(\%)$ & $17(58.6)$ & $7490(60.7)$ & $68(63.6)$ \\
\hline Platelet count $\left(10^{9} / \mathrm{L}\right)$, mean $^{\mathrm{b}}$ (range) & $249(153-301)$ & $235(175-309)$ & $229(182-285)$ \\
\hline Missing, $n(\%)$ & $16(55.2)$ & $4793(38.8)$ & $54(50.5)$ \\
\hline \multicolumn{4}{|l|}{ Clinical management } \\
\hline \multicolumn{4}{|l|}{ Documented antineoplastic use, $n(\%)$} \\
\hline Yes & $16(55.2)$ & $9422(76.3)$ & $71(66.4)$ \\
\hline No & $13(44.8)$ & $2927(23.7)$ & $36(33.6)$ \\
\hline Antineoplastic on or before CGP report date, $n(\%)$ & $12(41.4)$ & $7220(58.5)$ & $52(48.6)$ \\
\hline Documentation of any PD-L1 therapy, $n(\%)$ & $1(3.5)$ & $1921(15.6)$ & $9(8.4)$ \\
\hline PD-L1 therapy on or before CGP report date, $n(\%)$ & 0 & $643(5.2)$ & $2(1.9)$ \\
\hline $\begin{array}{l}\text { Time from first antineoplastic therapy to CGP report } \\
\text { (months), mean (range) }\end{array}$ & $1.03(0.31-7.28)$ & $3.84(0.07-17.90)$ & $5.36(-0.30-26.30)$ \\
\hline Follow-up time from CGP report (months), mean (range) & $7.49(2.23-12.50)$ & $5.72(1.74-13.50)$ & $7.56(2.22-16.20)$ \\
\hline
\end{tabular}

CGP comprehensive genomic profiling, ECOG Eastern Cooperative Oncology Group, NOS not otherwise specified, NTRK neurotrophic tyrosine receptor kinase, $P D-L 1$ programmed death-ligand 1

${ }^{a}$ Last ECOG performance status reported in 90-day period ending on CGP report date

${ }^{\mathrm{b}}$ Latest measurement within 90 days on or before CGP report date

Fig. 1 Study schema. $F M$ Foundation Medicine, NGS next-generation sequencing, NTRK neurotrophic tyrosine receptor kinase

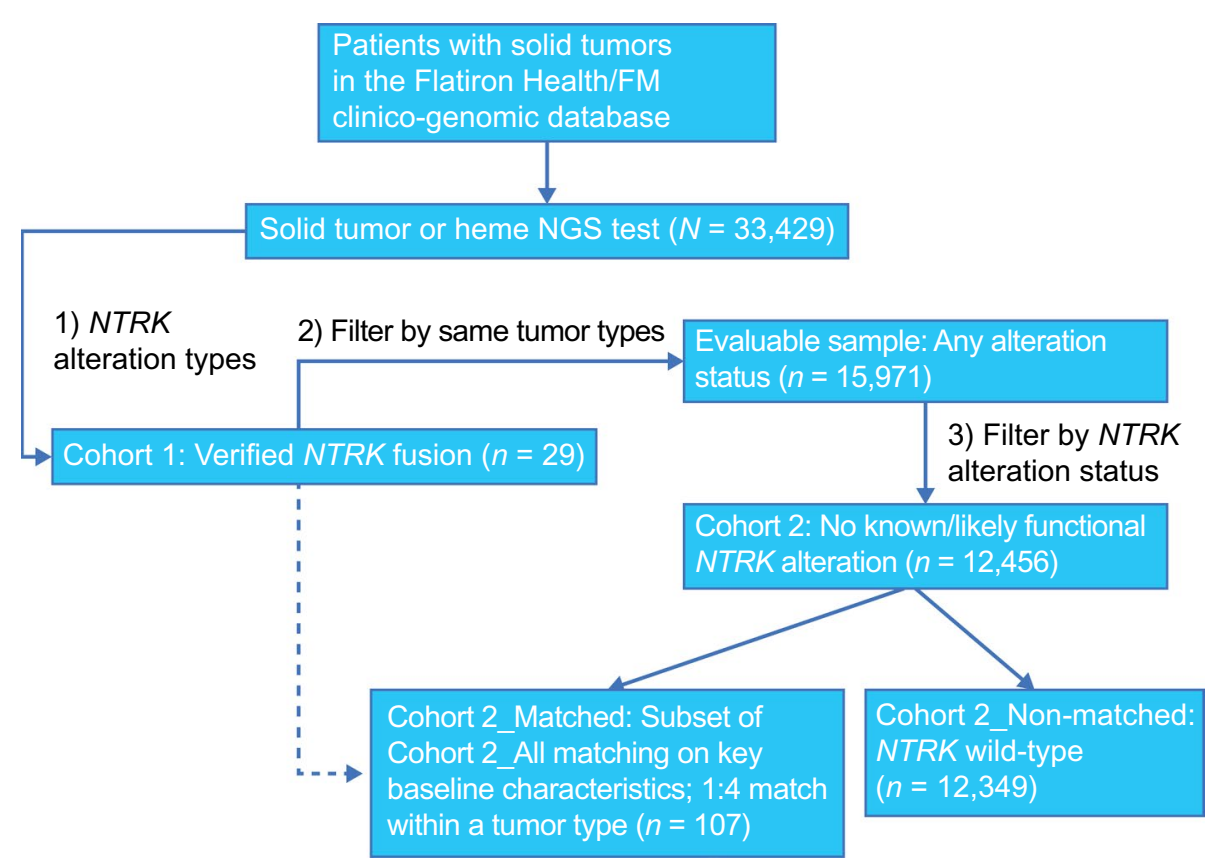

subset. The most frequently occurring biomarker reported in the non-matched group was KRAS alteration, reported in 4811 patients $(39 \%)$, followed by $E G F R$ alteration, reported in 956 patients $(8 \%)$. High TMB was reported in 654 patients (5\%). In the matched subset, KRAS alteration was reported most frequently $(n=25,23 \%)$, while the second most common was by $B R A F$ alteration $(n=8,7 \%)$. High TMB was reported in $3 \%$ of patients $(n=3$; Table 3$)$.

\section{Discussion}

Information on the natural history and genomic context of TRK fusion cancer is limited. To contextualize the activity observed in single-arm studies of TRK inhibitors in this disease, it is critical to understand whether NTRK gene fusions have an independent prognostic effect. This retrospective study investigated the characteristics and 
Table 2 NTRK gene partners

\begin{tabular}{llll}
\hline Fusion gene partner, $n$ & $\begin{array}{l}\text { NTRK1 } \\
(n=18)\end{array}$ & $\begin{array}{l}\text { NTRK2 } \\
(n=3)\end{array}$ & $\begin{array}{l}\text { NTRK3 } \\
(n=8)\end{array}$ \\
\hline$A C O 1^{\text {a }}$ & - & 1 & - \\
$A R H G E F 11^{\text {a }}$ & 1 & - & - \\
$D A B 2 I P$ & - & 1 & - \\
$E R C 1^{\text {a }}$ & 1 & - & - \\
$E T V 6$ & - & - & 8 \\
$F L V C R 1^{\text {a }}$ & 1 & - & - \\
GRIPAP1 & 1 & - & - \\
$I R F 2 B P 2$ & 1 & - & - \\
$M T A 1^{\text {a }}$ & 1 & - & - \\
$P D I A 3^{\mathrm{a}}$ & 1 & - & - \\
$R C S D 1^{\mathrm{a}}$ & 1 & - & - \\
$S Q S T M 1$ & - & 1 & - \\
$T P M 1$ & 1 & - & - \\
$T P M 3$ & 6 & - & - \\
$T P R$ & 3 & - & \\
\hline
\end{tabular}

NTRK neurotrophic tyrosine receptor kinase

${ }^{a}$ Fusion partners that have not been previously reported in the published literature

OS outcomes of matched real-world cohorts of patients with and without $N T R K$ gene fusions. The frequency of co-occurrence of NTRK gene fusions with other genomic biomarkers using a clinico-genomic database comprising clinical data from electronic health records linked to CGP data obtained during routine clinical care was also investigated. These evaluations were performed across all available tumor types, primarily owing to small sample sizes of NTRK gene fusions within each tumor type.

While no evidence for differences in survival was detected, there was a numerically shorter point estimate of median OS in the NTRK fusion cohort (12.5 months) compared with the NTRK wild-type cohort (16.5 months [HR 1.44; 95\% CI 0.61-3.37; $p=0.648]$ ]). Considering that this study was conducted before the approval of TRK inhibitors, no patients with NTRK gene fusion tumors had received TRK inhibitors (although one patient had received an unknown investigational agent in a clinical trial). Therefore, our findings reflect the natural history of tumors with $N T R K$ gene fusions. Further studies should be conducted to determine if there is a difference in survival between patients with $N T R K$ gene fusions vs $N T R K$ wild-type cancer.

Co-occurrence of oncogenic alterations in $A L K, B R A F$, $E R B B 2, E G F R, R O S 1$, and KRAS was uncommon in patients with $N T R K$ gene fusions, supporting the hypothesis that $N T R K$ gene fusions are the primary oncogenic drivers in tumors that harbor them. High TMB and high MSI were both more frequent in patients with NTRK gene fusions than in those without. Based on previous studies of TRK fusion cancer, the high TMB and high MSI observed in patients with NTRK gene fusions in our study is likely because of the high proportion of patients with MSI-high colorectal cancer in the NTRK fusion cohort. High MSI has been previously associated with the acquisition of kinase fusions in
Fig. 2 Kaplan-Meier plot for overall survival analysis using the comprehensive genomic profiling (CGP) report date as the index date (matched). $C I$ confidence interval, $H R$ hazard ratio, $N E$ not evaluable, NTRK neurotrophic tyrosine receptor kinase, $O S$ overall survival. ${ }^{\text {a }}$ wo patients with salivary gland secretory carcinoma in the NTRK fusion cohort were excluded from the OS analysis because of a lack of patients with this tumor type in the NTRK wild-type cohort

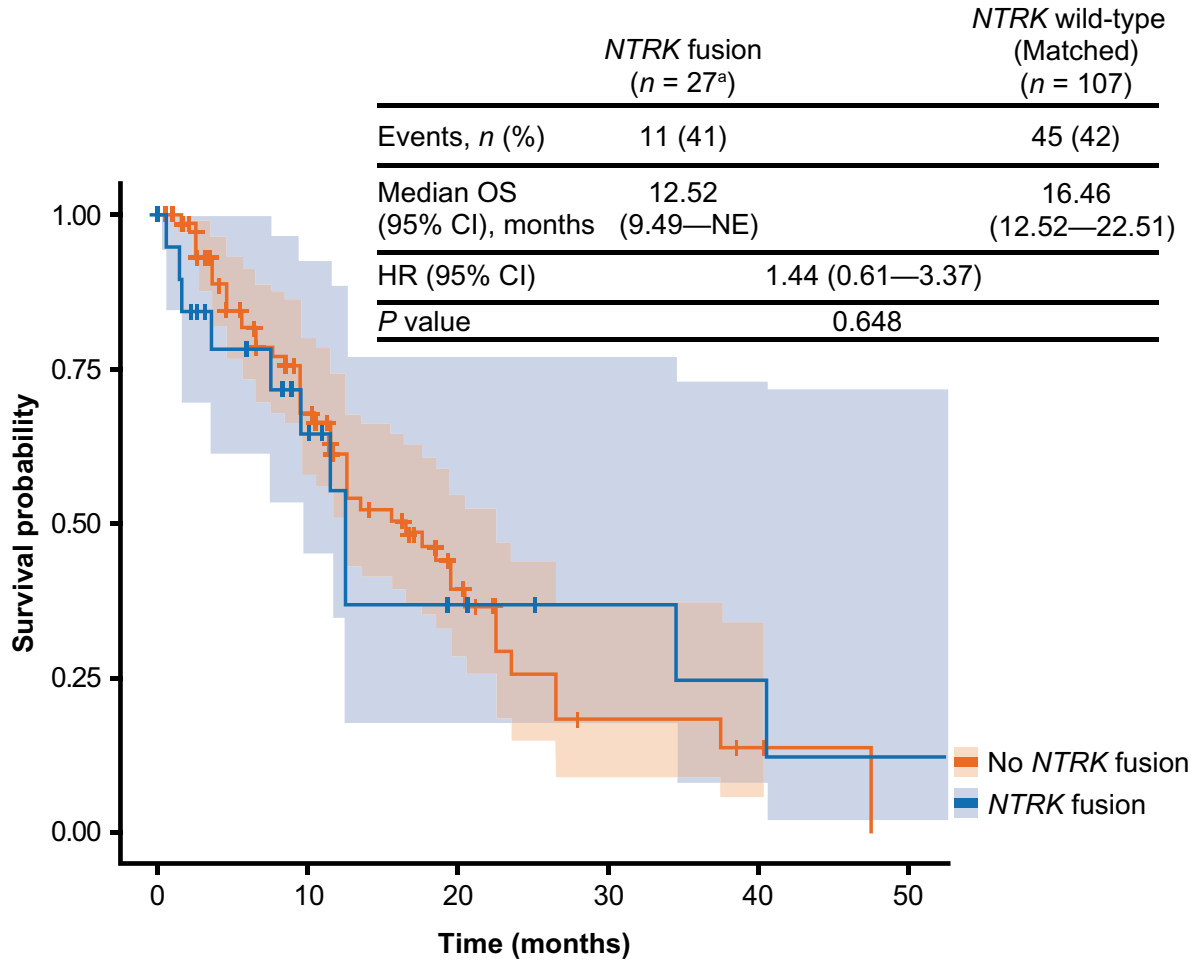


Table 3 Co-occurring biomarkers and molecular characteristics

\begin{tabular}{llll}
\hline & $\begin{array}{l}\text { Cohort } 1: \text { NTRK fusion } \\
(n=29)\end{array}$ & $\begin{array}{l}\text { Cohort 2 (non-matched): NTRK wild- } \\
\text { type }(n=12,349)\end{array}$ & $\begin{array}{l}\text { Cohort 2 (matched): } \\
\text { NTRK wild-type } \\
(n=107)\end{array}$ \\
\hline $\begin{array}{l}\text { Co-occurring biomarkers, }{ }^{\mathrm{a}} n(\%) \\
\text { TMB status }\end{array}$ & & & \\
High $(\geq 20 \mathrm{mut} / \mathrm{mB})$ & $6(20.7)$ & $654(5.3)$ & $3(2.8)$ \\
Medium $(<20, \geq 5.5 \mathrm{mut} / \mathrm{mB})$ & $3(10.3)$ & $3223(26.1)$ & $16(15.0)$ \\
MSI-high & $3 / 17^{\mathrm{b}}(17.6)$ & $93 / 7902^{\mathrm{b}}(1.2)$ & $1 / 59^{\mathrm{b}}(1.7)$ \\
$A L K$ rearrangement & 0 & $159(1.3)$ & $3(2.8)$ \\
$B R A F$ alteration & $1(3.5)$ & $812(6.6)$ & $8(7.5)$ \\
$E R B B 2$ amplification & 0 & $468(3.8)$ & $2(1.9)$ \\
$E G F R$ alteration & $1(3.5)$ & $956(7.7)$ & $6(5.6)$ \\
$R O S 1$ alteration & 0 & $90(0.7)$ & 0 \\
$K R A S$ alteration & $3(10.3)^{\mathrm{c}}$ & $4811(39.0)$ & $25(23.4)$ \\
\hline
\end{tabular}

MSI microsatellite instability, $N T R K$ neurotrophic tyrosine receptor kinase, TMB tumor mutation burden

"Variants of "known" or "likely" functional status were included, "ambiguous" and "unknown" were excluded

${ }^{\mathrm{b}}$ MSI status missing for 12 patients in Cohort 1, 4447 patients in Cohort 2 (non-matched), and 48 patients in Cohort 2 (matched)

${ }^{\mathrm{c}}$ One patient was subsequently reclassified as NTRK rearrangement rather than fusion

patients with colorectal cancer [17, 29]. The high proportion of patients with TRK fusion colorectal cancer in our study was unexpected given the low rate of kinase fusions in colorectal cancer overall [29]. Our observation reflects the high overall incidence of patients with colorectal cancer within the CGDB, which may be due to a high rate of molecular testing in colorectal cancer clinical practice.

There are some limitations with this analysis. This is a retrospective study covering a period of over 7 years during which the design of clinically available CGP assays was updated to improve sensitivity of detection for oncogenic gene fusions; therefore, there could be potential misclassifications (false negatives) in the NTRK wildtype cohort, and this study most likely underestimated the number of patients with NTRK gene fusions. Detection of gene fusions involving NTRK2 and NTRK3 may have been limited by DNA NGS coverage of the large intronic regions of these two genes [30]. As RNA-based testing focuses on coding sequences rather than introns [30], using RNA NGS may have enabled more sensitive detection of NTRK gene fusions in NTRK2 and NTRK3 and reduced the potential for false negatives. The study relied on structured electronic health record data to obtain clinical information, thus some important clinical variables that would require chart review, such as date of diagnosis, stage, exposure to certain therapies or study drugs, and endpoints (e.g., treatment response, progression), were not included. In addition, ECOG performance status was missing for a substantial portion of patients and matching on missing baseline ECOG performance status is less likely to have effectively balanced prognostic characteristics than matching on known baseline ECOG performance status. It was also not possible to use chart-confirmed index dates (e.g., a date of advanced diagnosis), which may be a more clinically meaningful index date than the index dates utilized in this study, or for delineating lines of therapy for patients. Treatments and outcomes that occurred outside of the clinic setting or were not documented in the patient's record were also not included. Therefore, the present study may have a degree of incompleteness and imprecision. To overcome limitations associated with the sparsity of available clinical data, one future solution could be to conduct a master observational study, a prospective observational study whereby patients are broadly included regardless of biomarker status, with extensive data collection performed for every patient. This new clinical study construct advances on master interventional study protocols such as the National Cancer Institute's Molecular Analysis for Therapy Choice Trial (NCI-MATCH) by hybridizing interventional protocols with comprehensive and standardized collection of real-world data [31, 32]. In addition, the ongoing non-interventional ON-TRK study of larotrectinib in patients with TRK fusion cancer will provide valuable insight into the real-world characteristics and outcomes of patients with NTRK gene fusions who receive larotrectinib [33].

Furthermore, the sample size was small overall and severely restricted within histologies, and thus conclusions about the OS of patients with NTRK fusion cancer compared to patients with NTRK wild-type cancer are limited. The differences in testing times during the patient's journey could also have impacted the interpretations of the OS results. A 


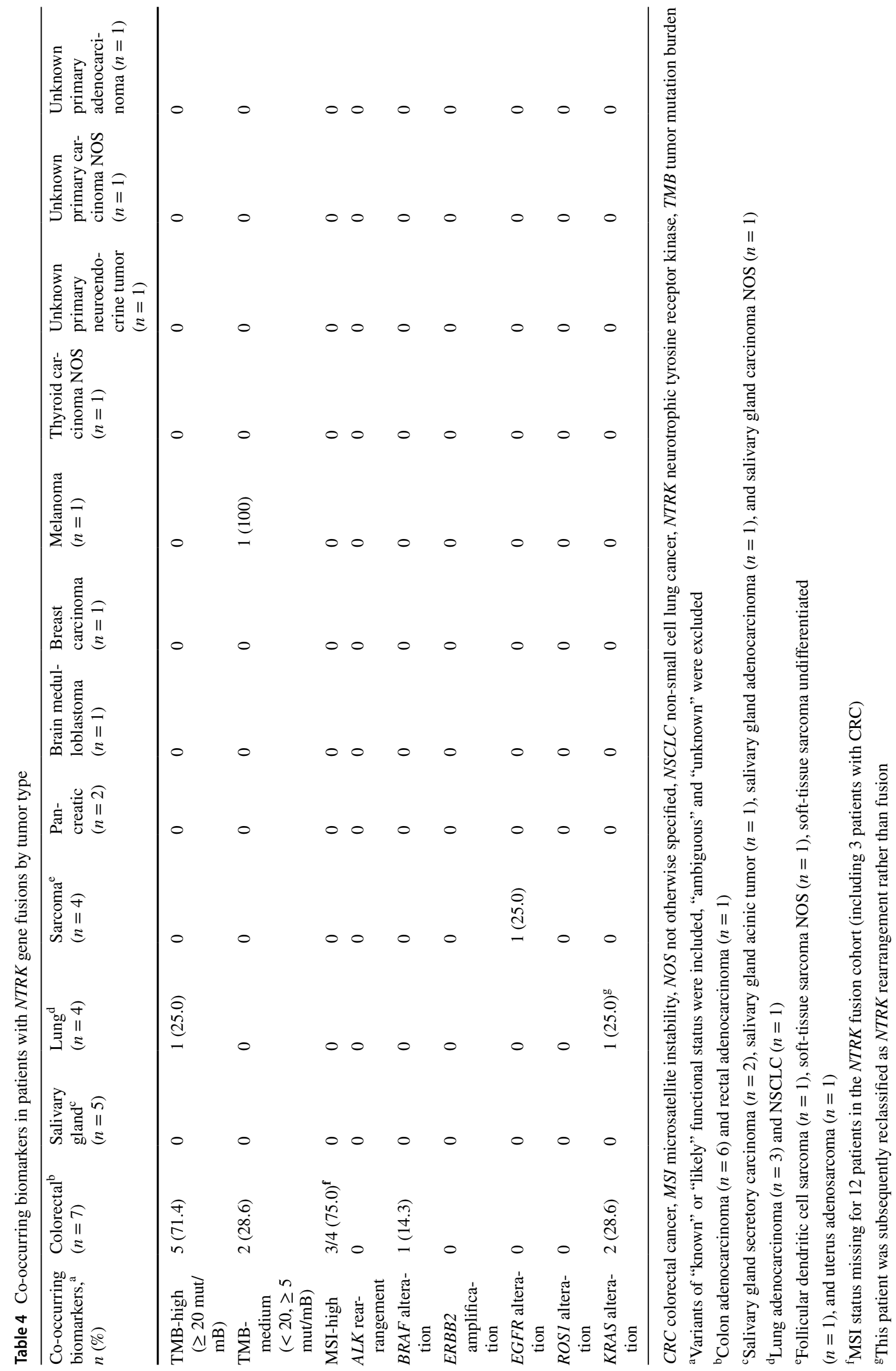


more recent and comprehensive study with a larger sample size would improve our understanding of this disease.

In summary, our study suggests that in patients with tumors harboring NTRK gene fusions, co-occurrence of other actionable biomarkers is generally uncommon, except for MSI-high in colorectal cancers [34, 35], supporting the hypothesis that NTRK gene fusions are the primary oncogenic drivers in tumors that harbor them. This highlights the importance of NTRK gene fusions as actionable drug targets and emphasizes the need for widespread adoption of broad panel genomic testing in routine oncology clinical practice. The study also demonstrated that patients with tumors that harbor an NTRK gene fusion may have an increased, or at least similar risk of death compared with the matched patients whose tumors are NTRK wild type, thus underscoring the potential clinical benefits of TRK inhibitor therapy for patients with TRK fusion cancer.

Supplementary Information The online version contains supplementary material available at https://doi.org/10.1007/s11523-021-00815-4.

Acknowledgements The authors thank the patients, their families, and all investigators involved in these studies. Medical writing support was provided by Farzana Miah, MSc, and editorial support was provided by Annabel Ola, MSc, all of Scion, London, supported by Bayer according to Good Publication Practice guidelines http://annals.org/aim/article/ 2424869/good-publication-practice-communicating-company-spons ored-medical-research-gpp3. The sponsor was involved in the study design, collection, analysis and interpretation of data, as well as data checking of information provided in the manuscript. However, ultimate responsibility for opinions, conclusions, and data interpretation lies with the authors.

\section{Declarations}

Funding This study was funded by Bayer HealthCare Pharmaceuticals, Inc.

Conflicts of interest/competing interests Lyudmila Bazhenova has served on advisory boards for Genentech, AstraZeneca, Boehringer Ingelheim, Takeda, and Blueprint. Xiaolong Jiao is an employee of Bayer HealthCare Pharmaceuticals, Inc. Andrew Lokker is an employee of Flatiron Health, Inc., an independent subsidiary of the Roche Group, and reports stock ownership in Roche and equity ownership in Flatiron Health, Inc. Jeremy Snider is an employee of Flatiron Health, Inc., an independent subsidiary of the Roche Group, and reports stock ownership in Roche and equity ownership in Flatiron Health, Inc. Emily Castellanos is an employee of Flatiron Health, Inc., an independent subsidiary of the Roche Group, and reports stock ownership in Roche and equity ownership in Flatiron Health, Inc. Virginia Fisher is an employee of Foundation Medicine, Inc., and reports stock ownership in Roche. Marc Fellous is an employee of Bayer HealthCare Pharmaceuticals, Inc. Shivani Nanda was an employee of Bayer HealthCare at the time the work was conducted. Jihong Zong and Karen Keating are employees of Bayer HealthCare.

Ethics approval This research study was conducted retrospectively from data obtained for clinical purposes in accordance with the 1964 Helsinki Declaration and its amendments.
Consent to participate Not applicable.

Consent for publication Not applicable.

Availability of data and material The data that support the findings of this study have been originated by Flatiron Health, Inc. and Foundation Medicine, Inc. These de-identified data may be made available upon request, and are subject to a license agreement with Flatiron Health and Foundation Medicine; interested researchers should contact cgdb-fmi@ flatiron.com to determine licensing terms.

Code availability Not applicable.

Author contributions All authors of this manuscript were involved in study design, data collection, or data analysis. All authors critically revised the manuscript and gave final approval of the submitted version.

Open Access This article is licensed under a Creative Commons Attribution-NonCommercial 4.0 International License, which permits any non-commercial use, sharing, adaptation, distribution and reproduction in any medium or format, as long as you give appropriate credit to the original author(s) and the source, provide a link to the Creative Commons licence, and indicate if changes were made. The images or other third party material in this article are included in the article's Creative Commons licence, unless indicated otherwise in a credit line to the material. If material is not included in the article's Creative Commons licence and your intended use is not permitted by statutory regulation or exceeds the permitted use, you will need to obtain permission directly from the copyright holder. To view a copy of this licence, visit http://creativecommons.org/licenses/by-nc/4.0/.

\section{References}

1. Amatu A, Sartore-Bianchi A, Siena S. NTRK gene fusions as novel targets of cancer therapy across multiple tumour types. ESMO Open. 2016;1(2):e000023.

2. Cocco E, Scaltriti M, Drilon A. NTRK fusion-positive cancers and TRK inhibitor therapy. Nat Rev Clin Oncol. 2018;15(12):731-47.

3. Indo Y, Tsuruta M, Hayashida Y, Karim MA, Ohta K, Kawano $\mathrm{T}$, et al. Mutations in the TRKA/NGF receptor gene in patients with congenital insensitivity to pain with anhidrosis. Nat Genet. 1996;13(4):485-8.

4. Greco A, Villa R, Fusetti L, Orlandi R, Pierotti MA. The Gly571Arg mutation, associated with the autonomic and sensory disorder congenital insensitivity to pain with anhidrosis, causes the inactivation of the NTRK1/nerve growth factor receptor. J Cell Physiol. 2000;182(1):127-33.

5. Klein R, Smeyne RJ, Wurst W, Long LK, Auerbach BA, Joyner $\mathrm{AL}$, et al. Targeted disruption of the trkB neurotrophin receptor gene results in nervous system lesions and neonatal death. Cell. 1993;75(1):113-22.

6. Xu B, Goulding EH, Zang K, Cepoi D, Cone RD, Jones KR, et al. Brain-derived neurotrophic factor regulates energy balance downstream of melanocortin-4 receptor. Nat Neurosci. 2003;6(7):736-42.

7. Yeo GSH, Connie Hung C-C, Rochford J, Keogh J, Gray J, Sivaramakrishnan $\mathrm{S}$, et al. A de novo mutation affecting human TrkB associated with severe obesity and developmental delay. Nat Neurosci. 2004;7(11):1187-9.

8. Huang EJ, Reichardt LF. Neurotrophins: roles in neuronal development and function. Annu Rev Neurosci. 2001;24:677-736. 
9. Vaishnavi A, Le AT, Doebele RC. TRKing down an old oncogene in a new era of targeted therapy. Cancer Discov. 2015;5(1):25-34.

10. Drilon A. TRK inhibitors in TRK fusion-positive cancers. Ann Oncol. 2019;30:viii23-30.

11. Bayer HealthCare Pharmaceuticals Inc. VITRAKVI prescribing information. 2018. http://www.labeling.bayerhealthcare.com/ html/products/pi/vitrakvi_PI.pdf. Accessed 6 Aug 2020.

12. European Medicines Agency. VITRKAVI SmPC. 2019. Available from: https://www.medicines.org.uk/emc/files/pil.10766.pdf. Accessed 27 Feb 2020.

13. Hong DS, DuBois SG, Kummar S, Farago AF, Albert CM, Rohrberg KS, et al. Larotrectinib in patients with TRK fusion-positive solid tumours: a pooled analysis of three phase $1 / 2$ clinical trials. Lancet Oncol. 2020;21(4):531-40.

14. Menichincheri M, Ardini E, Magnaghi P, Avanzi N, Banfi P, Bossi R, et al. Discovery of entrectinib: a new 3-aminoindazole as a potent anaplastic lymphoma kinase (ALK), c-ros oncogene 1 kinase (ROS1), and pan-tropomyosin receptor kinases (Pan-TRKs) inhibitor. J Med Chem. 2016;59(7):3392-408.

15. Roche AG. Rozlytrek prescribing information. 2019. https://www. gene.com/download/pdf/rozlytrek_prescribing.pdf. Accessed 18 Dec 2019.

16. Doebele RC, Drilon A, Paz-Ares L, Siena S, Shaw AT, Farago AF, et al. Entrectinib in patients with advanced or metastatic NTRK fusion-positive solid tumours: integrated analysis of three phase 1-2 trials. Lancet Oncol. 2020;21(2):271-82.

17. Rosen EY, Goldman DA, Hechtman JF, Benayed R, Schram AM, Cocco E, et al. TRK fusions are enriched in cancers with uncommon histologies and the absence of canonical driver mutations. Clin Cancer Res. 2020;26(7):1624-32.

18. Singal G, Miller PG, Agarwala V, Li G, Kaushik G, Backenroth $\mathrm{D}$, et al. Association of patient characteristics and tumor genomics with clinical outcomes among patients with non-small cell lung cancer using a clinicogenomic database. J Am Med Assoc. 2019;321(14):1391-9.

19. Frampton GM, Fichtenholtz A, Otto GA, Wang K, Downing $\mathrm{SR}, \mathrm{He} \mathrm{J}$, et al. Development and validation of a clinical cancer genomic profiling test based on massively parallel DNA sequencing. Nat Biotechnol. 2013;31(11):1023-31.

20. Fabrizio D, Milbury C, Yip W-K, Yilmazel B, Bai X, Liao J, et al. Clinical and analytic validation of FoundationOne $\mathrm{CDx}^{\mathrm{TM}}$ for NTRK fusion-positive solid tumors in patients treated with entrectinib [abstract A028]. Mol Cancer Ther. 2019;18(12 Suppl.):A028-A.

21. US Food and Drug Administration. FDA approves companion diagnostic to identify NTRK fusions in solid tumors for Vitrakvi. 2020. https://www.fda.gov/drugs/fda-approves-companion-diagn ostic-identify-ntrk-fusions-solid-tumors-vitrakvi. Accessed 22 Jan 2021.

22. Foundation Medicine. Our testing portfolio. 2021. https://www. foundationmedicine.com/portfolio. Accessed 28 Jan 2021.

23. Mahalanobis P. On the generalised distance in statistics. Proc Natl Inst Sci India. 1936;2(2):49-55.

24. Stuart EA. Matching methods for causal inference: a review and a look forward. Stat Sci. 2010;25(1):1-21.

25. Stuart EA, Lee BK, Leacy FP. Prognostic score-based balance measures can be a useful diagnostic for propensity score methods in comparative effectiveness research. J Clin Epidemiol. 2013;66(8 Suppl):S84-90.e1.

26. Austin PC. An introduction to propensity score methods for reducing the effects of confounding in observational studies. Multivar Behav Res. 2011;46(3):399-424.

27. Curtis MD, Griffith SD, Tucker M, Taylor MD, Capra WB, Carrigan G, et al. Development and validation of a high-quality composite real-world mortality endpoint. Health Serv Res. 2018;53(6):4460-76.

28. El-Deiry WS, Goldberg RM, Lenz HJ, Shields AF, Gibney GT, Tan AR, et al. The current state of molecular testing in the treatment of patients with solid tumors. 2019. CA Cancer J Clin. 2019;69(4):305-43.

29. Cocco E, Benhamida J, Middha S, Zehir A, Mullaney K, Shia J, et al. Colorectal carcinomas containing hypermethylated MLH1 promoter and wild type BRAF/KRAS are enriched for targetable kinase fusions. Cancer Res. 2019;79(6):1047-53.

30. Penault-Llorca F, Rudzinski ER, Sepulveda AR. Testing algorithm for identification of patients with TRK fusion cancer. J Clin Pathol. 2019;72(7):460-7.

31. Dickson D, Johnson J, Bergan R, Owens R, Subbiah V, Kurzrock $\mathrm{R}$. The Master observational trial: a new class of master protocol to advance precision medicine. Cell. 2020;180(1):9-14.

32. Flaherty KT, Gray R, Chen A, Li S, Patton D, Hamilton SR, et al. The molecular analysis for therapy choice (NCI-MATCH) trial: lessons for genomic trial design. J Natl Cancer Inst. 2020;112(10):1021-9.

33. Leyvraz S, Yang JCH, Casali PG, Castro G, Kim ES, Lassen UN, et al. 604TiP ON-TRK: a non-interventional study of larotrectinib in patients with TRK fusion cancer. Ann Oncol. 2020;31:S503.

34. Bonneville R, Krook MA, Kautto EA, Miya J, Wing MR, Chen $\mathrm{HZ}$, et al. Landscape of microsatellite instability across 39 cancer types. JCO Precis Oncol. 2017;2017:PO.17.00073.

35. Chou A, Fraser T, Ahadi M, Fuchs T, Sioson L, Clarkson A, et al. NTRK gene rearrangements are highly enriched in MLH1/PMS2 deficient, BRAF wild-type colorectal carcinomas-a study of 4569 cases. Mod Pathol. 2020;33:924-32. 